\title{
Agonist and Antagonist Actions of Buprenorphine on Three Types of Opioid Receptor in Isolated Preparations
}

\author{
Midori KAJIWARA, Kazuko AOKI, Kaori ISHII, Hiroaki NUMATA, \\ Teruhiko MATSUMIYA and Tetsuo OKA* \\ Department of Pharmacology, School of Medicine, Tokai University, \\ Isehara 259-11. Japan
}

Accepted September 20, 1985

\begin{abstract}
Both agonist and antagonist actions of buprenorphine on isolated preparations were studied. The $\mathrm{K}_{\mathrm{e}}$ (equilibrium dissociation constant) values of both naloxone and $\operatorname{Mr} 2266$ [(-)-2-(3-furylmethyl)-5,9-diethyl-2'-hydroxy-6,7benzomorphan] against buprenorphine and the ratio of IC50 (concentration of the drug to produce $50 \%$ inhibition of the twitch) value of buprenorphine after to before exposure of mouse vas deferens to $\beta$-FNA ( $\beta$-fumaramate methyl ester derivatives of naltrexone), an irreversible mu antagonist, suggest that buprenorphine acts as both a mu and kappa agonist on mouse vas deferens. The agonist effect of buprenorphine at relatively high doses on guinea-pig ileum and mouse vas deferens and the negative agonist effect on both rat and rabbit vas deferens indicate that buprenorphine acts as a partial agonist on isolated preparations. The $\mathrm{K}_{\mathrm{e}}$ values of buprenorphine show that buprenorphine has about equal antagonist effectiveness against a mu and kappa agonist with approximately five-fold lower effectiveness against a delta agonist. The possible mechanisms for the several characteristic actions of buprenorphine on guinea-pig ileum such as the slow onset of action, the increased magnitude of inhibition after washing the tissue, the negative elimination of the inhibition by either washing the tissue or the naloxone administration, and the negative elimination of the antagonist action by washing the tissue were discussed.
\end{abstract}

Buprenorphine, a semisynthetic opioid derived from the opium alkaloid thebaine, is a highly lipophilic oripavine derivative containing a cyclopropylmethyl substitution (1). It is a clinically effective analgesic that is 25 to 50 times more potent than morphine and produces little if any physical dependence of clinical significance (2). Thus, buprenorphine seems to be a clinically interesting analgesic of low abuse potential. However, its choice of opioid receptor subtype as an agonist is controversial. Martin et al. (3) have indicated that buprenorphine acts as a partial agonist on mu receptors in the chronic spinal dog. In contrast. Tyers (4) who has studied a number of opioids in a variety of antinociceptive and other tests in rodents, has suggested that

* To whom all correspondence should be addressed. buprenorphine's agonist actions are primarily at kappa receptors. The purpose of the present investigation, therefore, was to determine the choice by buprenorphine of opioid-receptor type in isolated preparations as an agonist. Additionally, antagonistic effectiveness of buprenorphine at each opioid-receptor type was also studied.

\section{Materials and Methods}

Chemicals: Gifts of compounds which were gratefully received were naloxone- $\mathrm{HCl}$ from Sankyo Company (Tokyo): Mr 2266 [(-)-2(3-furylmethyl)-5,9-diethyl-2' - hydroxy-6,7benzomorphan] from Nippon C.H. Boehringer Sohn Co., Ltd. (Osaka); ethylketocyclazocine from Sterling-Winthrop Res. Inst. (Rensselaer New York, U.S.A.) ; buprenorphine- $\mathrm{HCl}$ from Otsuka Pharmaceutical Co., Ltd, (Tokyo); 
$\left[D-A_{a}{ }^{2}, D-L_{e}{ }^{5}\right]$-enkephalin from Dr. M. Ueki, Science University of Tokyo (Tokyo) and $\beta$-FNA ( $\beta$-fumaramate methyl ester derivative of naltrexone) from Dr. J.W. Holaday, Walter Reed Army inst. Res. (Washington, D.C., U.S.A.). Morphine-HCl was purchased from Takeda Chemical Ind., Ltd. (Osaka).

In vitro isolated preparations: Male ICP$\mathrm{JCL}$ mice weighing 30-40 g, male Hartley guinea-pigs weighing $300-500 \mathrm{~g}$, male Wistar rats weighing $200-300 \mathrm{~g}$, and male Japan White rabbits weighing $2.6-3.8 \mathrm{~kg}$ were used for this study. The myenteric plexus-longitudinal muscle strip of guineapig ileum and the mouse, rat or rabbit vas deferens were prepared, and the preparations were set up for electrical stimulation as described previously (5). The \% inhibition of the stimulated muscle twitch produced by a drug was plotted against the log concentration of the drug to estimate the IC50 (concentration of the drug to produce 50\% inhibition of the twitch). The $K_{e}$ (equilibrium dissociation constant) values of opioid antagonists against opioid agonists were determined by the 'single' dose method of Kosterlitz and Watt (6).

\section{Results}

Agonist action on isolated preparations: The inhibitory action on the electricallyevoked contractions of guinea-pig ileum of buprenorphine was slow in onset and gradually reached its maximum, when compared with that of either morphine or ethylketocyclazocine (Fig. 1). Interestingly, when the tissue was washed before the buprenorphine-induced inhibition reached its maximum, the magnitude of the inhibition was increased after washing the tissue (Fig. 2). Additionally, Fig. 2 shows that the buprenorphine-induced inhibition relatively rapidly reached its maximum when high doses were administered. Moreover, Fig. 2 shows that the buprenorphine-induced inhibition could not be eliminated by either the vigorous washing for three hours or the administration of an opioid antagonist, naloxone. When naloxone was given before the buprenorphine administration, however, it could antagonize the inhibition produced by buprenorphine (Fig. 3). Interestingly, naloxone, which was generally believed to be a pure antagonist, produced slight inhibition of the contractions by itself (Fig. 3), although the dose employed was quite high. When the tissue was washed repeatedly after the administration of naloxone and buprenorphine in this order, however, the inhibition of contractions appeared (Fig. 3 ), indicating that naloxone was eliminated, but buprenorphine was not eliminated by washing the tissue and seemed to stay on the opioid receptors.

Both the IC50 value of buprenorphine and the $K_{e}$ value of antagonists against buprenorphine in guinea-pig ileum could not be estimated since the dose-effect curve of buprenorphine could not be constructed due to negative elimination of the inhibition by washing the tissue. However, as Fig. 1 shows, buprenorphine was approximately 100-fold more potent than morphine, but four-fold less potent than ethylketocyclazocine to inhibit the contractions of guinea-pig ileum. Additionally, as shown in Figs. 1 and 2. buprenorphine acted as a partial agonist on guinea-pig ileum since at doses ten-fold

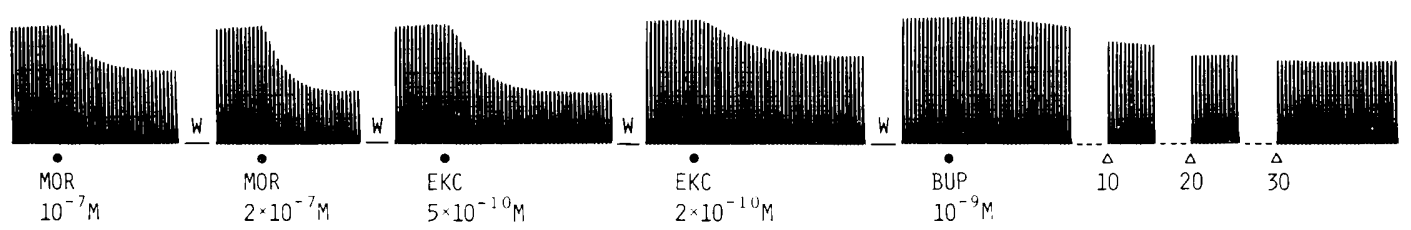

Fig. 1. The inhibitory potency of buprenorphine (BUP) relative to that of either morphine (MOR) or ethylketocyclazocine (EKC) in the electrically-evoked contractions of guinea-pig ileum. An opioid was added to the bath at the dot. After the opioid-induced inhibition reached its maximum, the tissue was washed $(W)$. Since the BUP-induced inhibition gradually reached its maximum, the records from 5 to 10,12 to 20 , and 22 to 30 minutes after the BUP administration were omitted (---). The numbers (10, 20 and 30) under the open triangle show the time in minutes after the BUP administration. 
higher than the IC50's, both morphine, a full mu agonist, and ethylketocyclazocine, a full kappa agonist, produced the complete inhibition of contractions of guinea-pig ileum. Moreover, Figs. 1 and 2 show that the dose-response curve of buprenorphine was not likely to be a bell-shaped one, but most likely to be flat.

In contrast to results shown in Fig. 2, in mouse vas deferens, the buprenorphineinduced inhibition of contractions could be

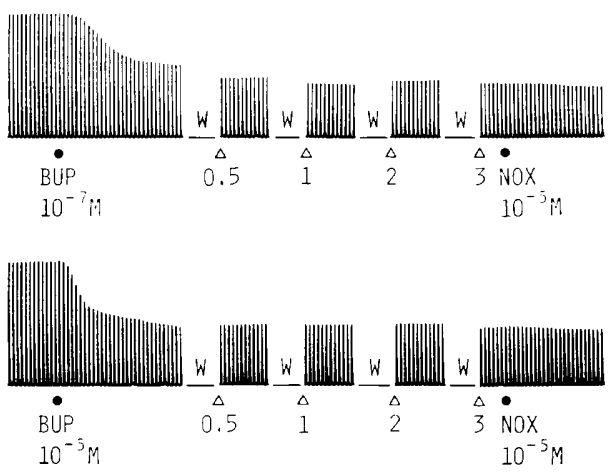

Fig. 2. The ceiling inhibitory effect of buprenorphine (BUP) on guinea-pig ileum and the negative elimination of the BUP-induced inhibition by either washing the tissue or the naloxone (NAL) administration. Either BUP or NAL was administered at the dot. The tissue was washed (W) five minutes after the BUP administration. Thereafter, the electricallyevoked contractions were recorded for five minutes starting from 0.5, 1, 2 and 3 hours after the BUP administration, although the records of contractions starting from 0.5, 1 and 2 hours were shown only for two minutes on the figure. The tissue was washed (W) repeatedly when contractions were not recorded. The numbers $(0.5,1,2$ and 3 ) under the open triangle show the time in hours after the BUP administration. reversed by naloxone even when an antagonist was given after the buprenorphine administration (Fig. 4). Additionally, the buprenorphine-produced inhibition could also be reversed by washing the tissue repeatedly for approximately two hours (Fig. 4). Thus, both the IC50 value of buprenorphine and the $K_{e}$ value of antagonists against buprenorphine in mouse vas deferens could be estimated. The inhibitory potency of buprenorphine relative to that of either morphine or ethylketocyclazocine in mouse vas deferens was approximately 67 or 0.69 . respectively (Table 1 ), showing that the relative potency of buprenorphine against either morphine or ethylketocyclazocine in mouse vas deferens was similar to that in guinea-pig ileum. Additionally, as shown in Figs. 4 and 5, buprenorphine acted as a partial agonist on mouse vas deferens since full agonists such as morphine and ethylketocyclazocine at doses ten-fold higher than

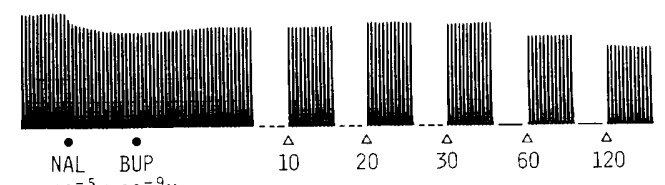

$$
10^{-5} \mathrm{M} 10^{-9} \mathrm{M}
$$

Fig. 3. The antagonism of buprenorphine (BUP) induced inhibition by naloxone (NAL) in guinea-pig ileum and the elimination of NAL but the negative elimination of BUP by washing the tissue. Either NAL or BUP was given at the dot. The records of contractions from 5 to 10,12 to 20 , and 22 to 30 minutes after the BUP administration were omitted $(--)$. The tissue was washed $(W)$ repeatedly from 32 to 50 and 65 to 110 minutes after the BUP administration. The numbers (10,20,30,60 and 120) under the open triangle show the time in minutes after the BUP administration.

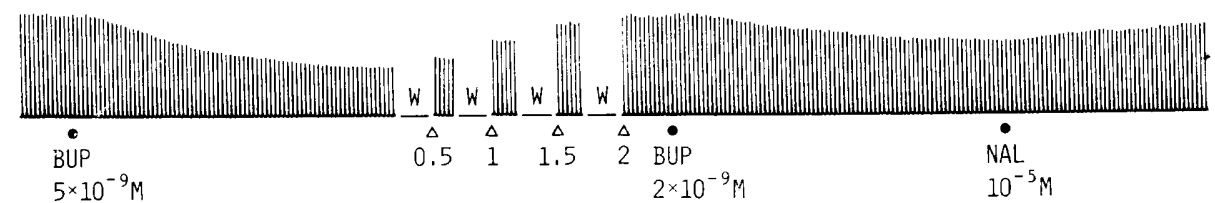

Fig. 4. The reversal of the buprenorphine (BUP)-induced inhibition by either washing the tissue (W) or naloxone (NAL) in mouse vas deferens. The tissue was washed repeatedly from 12 to 20,32 to 50 , 62 to 80 and 92 to 110 minutes after the BUP administration. Either BUP or NAL was given at the dot. The numbers $(0,5,1,1.5$ and 2$)$ under the open triangle show the time in hours after the BUP administration. 
Table 1. The potency of buprenorphine relative to that of either morphine or ethylketocyclazocine in mouse vas deferens

\begin{tabular}{lccccc}
\hline Opioids & \multicolumn{2}{c}{ IC50 $(\mathrm{nM})$} & \multicolumn{2}{c}{ Relative potency } \\
& Group 1 & Group 2 & Group 1 & Group 2 \\
Buprenorphine & $30.7 \pm 17$ & $9.65 \pm 2.6$ & $66.9 \pm 8.0$ & $0.686 \pm 0.082$ \\
Morphine & $1530 \pm 570$ & - & 1 & - \\
Ethylketocyclazocine & & $6.06 \pm 1.7$ & - & 1 \\
\hline
\end{tabular}

Each value represents the mean \pm S.E. of 5 experiments. Since each preparation has its own sensitivity to an opioid as shown in the data of IC50 values of buprenorphine, and the inhibition produced by either buprenorphine, morphine or ethylketocyclazocine was relatively difficult to eliminate by washing the tissue, the experiment was carried out using two separate groups.

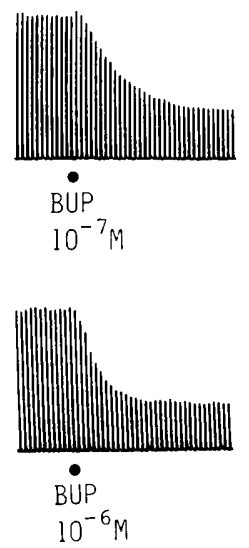

Fig. 5. The ceiling inhibitory effect of buprenorphine (BUP) on the electrically-evoked contractions of mouse vas deferens. BUP was given at the dot.

IC50 completely inhibited the contractions of mouse vas deferens. The $K_{e}$ value of $\mathrm{Mr}$ 2266 against buprenorphine was similar to that against either morphine or ethylketocyclazocine, but significantly different from that against $\left[D-A l a^{2}, D-L_{e}{ }^{5}\right]$-enkephalin (Table 2), indicating that buprenorphine did not act as a delta agonist, but acted as either a mu or kappa agonist. However, the $K_{e}$ value of naloxone against buprenorphine was higher than that against morphine and lower than that against ethylketocyclazocine (Table 2), suggesting that buprenorphine acted on both mu and kappa receptors as an agonist. Additionally, the ratio of the IC50 value of buprenorphine after to before exposure of mouse vas deferens to $\beta$-FNA, which had been reported to be an irreversible mu antagonist (7), was 2.66 (Table 3), also indicating that buprenorphine acted not only on mu receptors but also on the other receptors as well. The ratio of the IC50 value of either morphine, ethylketocyclazocine, or $\left[D-A l a^{2}, D-L e u^{5}\right]$-enkephalin in the presence of $\beta$-FNA to that in the absence of $\beta$-FNA was $5.0,1.1$ or 1.0 , respectively (Table 3 ), showing that $\beta$-FNA was an irreversible mu antagonist and consistent with the previous report (7).

In contrast to guinea-pig ileum and mouse vas deferens, buprenorphine at doses ranging from $10^{-8}$ to $10^{-5} \mathrm{M}$ had no agonist action at all on both rat and rabbit vas deferens.

Antagonist action on isolated preparations: Since the agonist action of buprenorphine was slow in onset and could not be eliminated readily by washing the tissue, the time course and the elimination of antagonistic effectiveness of buprenorphine were studied. Buprenorphine had no antagonist action against either morphine and ethyketocyclazocine in guinea-pig ileum or [D-Ala², $D$-Leu ${ }^{5}$-enkephalin in mouse vas deferens when agonists were administered three minutes after the buprenorphine administration (Table 4). When agonists were given 30 minutes after the buprenorphine administration, however, buprenorphine showed potent antagonist action, the mean \pm S.E. of the $K_{e}$ values of buprenorphine in four experiments against morphine and ethylketocyclazocine in guinea-pig ileum or [DAla ${ }^{2}$, D-Leu ${ }^{5}$-enkephalin in mouse vas deferens being $1.11 \pm 0.37$ and $1.08 \pm 0.41$ or $4.84 \pm 1.1 \mathrm{nM}$, respectively. Additionally, when the tissue was washed repeatedly after the effect of the initial agonist reached its 
Table 2. The Ke values of naloxone and Mr 2266 against buprenorphine, morphine, ethylketocyclazocine and [D-Ala $\left.{ }^{2}, \mathrm{D}-\mathrm{Leu}^{5}\right]$-enkephalin in the mouse vas deferens

\begin{tabular}{|c|c|c|}
\hline \multirow{2}{*}{ Opioids } & \multicolumn{2}{|c|}{$\mathrm{Ke}(\mathrm{nM})$} \\
\hline & Naloxone & Mr 2266 \\
\hline Buprenorphine & $10.6 \pm 1.3$ & $4.23 \pm 0.38$ \\
\hline Morphine & $4.11 \pm 0.51$ & $4.02 \pm 0.56$ \\
\hline Ethylketocyclazocine & $15.2 \pm 1.7$ & $3.95 \pm 0.49$ \\
\hline$\left[D-A l a^{2}, D-L^{2} u^{5}\right]-E n k e p h a l i n$ & $25.6 \pm 2.4$ & $23.7 \pm 3.1$ \\
\hline
\end{tabular}

Each value represents the mean \pm S.E. of 4 experiments.

Table 3. The IC50 ratio of mu-, kappa- and delta-agonist, and buprenorphine in mouse vas deferens

\begin{tabular}{ll}
\hline Opioids & IC50 ratio \\
\hline Morphine & $5.0 \pm 0.5$ \\
Ethylketocyclazocine & $1.1 \pm 0.1$ \\
{$\left[D-A l a^{2}\right.$, D-Leu ${ }^{5}$-Enkephalin } & $1.0 \pm 0.1$ \\
Buprenorphine & $2.66 \pm 0.2$ \\
\hline
\end{tabular}

After exposure of mouse vas deferens to $\beta$-FNA for 30 minutes at a concentration of $10^{-8} \mathrm{M}$, the tissue was repeatedly washed before the agonist administration. Each value represents the mean $\pm S$.E. of 4 experiments. a $C 50$ ratio represents the ratio of the 1 C50 value of an agonist after to before exposure of mouse vas deferens to $\beta$-FNA.

Table 4. The time course and the elimination or negative elimination of antagonistic effectiveness of buprenorphine against mu- and kappa-agonist in guinea-pig ileum (GPI) and that against delta-agonist in mouse vas deferens (MVD)

\begin{tabular}{cccc}
\hline \multirow{2}{*}{$\begin{array}{c}\text { Minutes after } \\
\text { buprenorphine }\end{array}$} & \multicolumn{3}{c}{ Ke (nM) } \\
\cline { 2 - 4 } & $\begin{array}{c}\text { Morphine } \\
\text { in GPI }\end{array}$ & $\begin{array}{l}\text { Ethylketocyclazocine } \\
\text { in GPI }\end{array}$ & $\begin{array}{c}\text { [D-Ala }{ }^{2} \text {, D-Leu }{ }^{5} \text { ]-Enkephalin } \\
\text { in MVD }\end{array}$ \\
\cline { 2 - 4 } 3 & No antagonism & No antagonism & No antagonism \\
30 & $0.921 \pm 0.18$ & $0.911 \pm 0.25$ & $8.46 \pm 1.3$ \\
60 & $0.869 \pm 0.17$ & $1.22 \pm 0.32$ & $19.1 \pm 4.1$ \\
90 & $0.755 \pm 0.11$ & $1.34 \pm 0.39$ & $40.4 \pm 3.4$ \\
120 & $1.02 \pm 0.27$ & $1.55 \pm 0.47$ & No antagonism \\
\hline
\end{tabular}

Antagonistic effectiveness of buprenorphine was estimated at 3, 30, 60, 90 and 120 minutes after its administration. The tissue was washed repeatedly between each estimation. Each value represents the mean \pm S.E. of 8 experiments in GPI and 4 experiments in MVD.

maximum and agonists were then given 30 minutes after the buprenorphine administration, buprenorphine still showed potent antagonist action against either a mu, kappa or delta agonist (Table 4), showing that an antagonist action as well as agonist action of buprenorphine was not eliminated readily by washing the tissue. The $\mathrm{K}_{\mathrm{e}}$ value of buprenorphine against morphine 30 minutes after the buprenorphine administration was not significantly different from that either 60,90 or 120 minutes after the buprenorphine administration in guinea-pig ileum, showing that the antagonist effectiveness as well as the agonist potency of buprenorphine was not decreased by washing the tissue for two hours. Similarly, the $\mathrm{K}_{\mathrm{e}}$ value against ethylketocyclazocine 30 minutes after the buprenorphine administration was not significantly different from that either 60,90 or 120 minutes after the buprenorphine administration in guinea-pig ileum. In contrast to 
the data in guinea-pig ileum, in mouse vas deferens, the antagonist effectiveness of buprenorphine was gradually decreased by washing the tissue and could not be detectable 120 minutes after the administration (Table 4). Thus, both the agonist and antagonist action of buprenorphine on mouse vas deferens could be eliminated by washing the tissue for two hours (Fig. 4 and Table 4).

The $K_{e}$ value of buprenorphine 30 minutes after the administration against morphine was not significantly different from that against ethylketocyclazocine, but approximately fivefold lower than that against [D-Ala², DLeu ${ }^{5}$ ]-enkephalin, indicating that the antagonist effectiveness of buprenorphine against a mu agonist was essentially the same as that against a kappa agonist, but was significantly higher than that against a delta agonist.

\section{Discussion}

The $K_{e}$ values of $\mathrm{Mr} 2266$ against buprenorphine in mouse vas deferens indicate that as an agonist, buprenorphine does not act on delta receptors but acts on either mu or kappa receptors since the $\mathrm{K}_{\mathrm{e}}$ values of $\mathrm{Mr}$ 2266 against either mu or kappa agonists and delta agonists have been shown to be 1-5 $\mathrm{nM}$ and 10-50 $\mathrm{nM}$, respectively $(5,8$, $9)$. On the other hand, the fact that the $K_{e}$ values of naloxone against buprenorphine lie between those against a mu agonist and those against a kappa agonist (5, 8, 9) suggests that buprenorphine acts on both mu and kappa receptors as an agonist. Additionally, the fact that the ratio of the IC50 value of buprenorphine after to before exposure of mouse vas deferens to $\beta$-FNA (IC50 ratio) is 2.66 indicates that buprenorphine acts not only on mu receptors but also on the other receptors as well, since the IC50 ratio of buprenorphine lies between that of a mu agonist and that of either a kappa or delta agonist. Thus, the $K_{e}$ value of either Mr 2266 or naloxone and the 1 C50 ratio strongly suggest that buprenorphine acts on both mu and kappa receptors as an agonist.

Data shown in Figs. 1,2 and 5 show that buprenorphine does not act as a full agonist but acts as a partial agonist on both guineapig ileum and mouse vas deferens, being consistent with data obtained with chronic spinal dogs by Martin et al. (3). Additionally. the fact that buprenorphine at doses ranging from $10^{-8}$ to $10^{-5} \mathrm{M}$ has no agonist action at all on both rat and rabbit vas deferens also suggests that buprenorphine is a partial agonist, since a partial kappa agonist, nalorphine, has no agonist activity in rabbit vas deferens, and a strong full mu agonist, FK 33-824 [Tyr-D-Ala-Gly-MePhe-Met(O)ol], significantly inhibits the contractions of rat vas deferens (T. Oka et al., unpublished data). However, the dose-response curve of buprenorphine at doses ranging from $10^{-9}$ to $10^{-5} \mathrm{M}$ in guinea-pig ileum or from $10^{-8}$ to $10^{-6} \mathrm{M}$ in mouse vas deferens seems not to be an inverted $U$, buito be a fiat one, although a bell-shaped dose-response curve of buprenorphine for the antinociceptive test in rodents has been reported $(10,11)$. The reason for the inconsistency existing between the antinociceptive action on rodents and the inhibitory action on contractions of the isolated preparation for the shape of the doseresponse curve of buprenorphine is not clear at present.

The $K_{e}$ value of buprenorphine shows that buprenorphine has about equal antagonist effectiveness against a mu and kappa agonist with about five-fold lower effectiveness for a delta agonist. Additionally, it shows that the antagonist effectiveness of buprenorphine is extremely high-between one and two orders of magnitude greater than that of naloxone $(5,8,9)$.

As shown in Figs. 1 and 2 and Table 4 , there are several characteristic actions of buprenorphine on guinea-pig ileum such as the slow onset of action (Fig. 1 and Table 4), the increased magnitude of inhibition after washing the tissue (Fig. 2), the negative elimination of the inhibition by either washing the tissue or the naloxone administration (Fig. 2 ), and the negative elimination of the antagonist action by washing the tissue (Table 4). It is difficult to interpret these characteristics of buprenorphine at present. However, one possible mode is the interaction of buprenorphine, which is dissolved in the lipid of the nerve cell membrane where opioid receptors exist, with opioid receptors. This mode is supported by the logical con- 
sideration that a large portion of the administered buprenorphine initially dissolves in the lipid of the cell membrane since buprenorphine is a highly lipophilic compound, and the surface area of the lipid portion of the cell membrane must be extremely larger than that of the specific binding sites of burenorphine on opioid receptor protein exposed to the extracellular fluid. A drawback of this hypothetical mode of action is the fact that there is no evidence about the interaction of the dissolved drug in the lipid with receptors. If the hypothesis is true, however, several characteristic actions of buprenorphine can be interpreted by this hypothetical mode of action. Additionally, the existing differences between guinea-pig ileum and mouse vas deferens with regards to the effects of both washing the tissue and naloxone on the buprenorphine-induced inhibition can be interpreted as caused by differences in the lipid composition of the nerve cell membrane. However, the precise mode of these peculiar but interesting actions of buprenorphine on isolated preparations remains to be elucidated.

\section{References}

1 Lewis, J.W.: Ring C-bridged derivatives of thebaine and oripavine. Adv. Biochem. Psychopharmacol. 8, 123-136 (1974)

2 Jasinski, D.R., Pevnick, J.S. and Griffith, J.D.: Human pharmacology and abuse potential of the analgesic buprenorphine. Arch. Gen. Psychiatry 35, 501-516 (1978)

3 Martin, W.R., Eades, D.G., Thompson, J.A., Huppler, R.E. and Gilbert, P.E.: The effects of morphine- and nalorphine-like drugs in the nondependent and morphine-dependent chronic spinal dog. J. Pharmacol. Exp. Ther. 197, 517532 (1976)

4 Tyers, M.B.: A classification of opiate receptors that mediate antinociception in animals. Br. J. Pharmacol. 69, 503-512 (1980)

5 Oka, T., Negishi, K., Suda, M., Sawa, A., Fujino, M. and Wakimasu, M.: Evidence that dynorphin$(1-13)$ acts as an agonist on opioid $\kappa$-receptors. Eur. J. Pharmacol. 77, 137-141 (1982)

6 Kosterlitz, H.W. and Watt, A.J.: Kinetic parameters of narcotic agonists and antagonists, with particular reference to $\mathrm{N}$-allylnoroxymorphone (naloxone). Br. J. Pharmacol. 33, 266276 (1968)

7 Takemori, A.E., Larson, D.L. and Portoghese, P.S.: The irreversible narcotic antagonist and reversible agonist properties of the fumarate methy! ester derivative of naltrexone. Eur. J. Pharmacol. 70, 445-451 (1981)

8 Oka, T., Negishi, K., Kajiwara, M., Watanabe, Y., Ishizuka, Y. and Matsumiya, T.: The choice of opiate receptor subtype by neo-endorphins. Eur. J. Pharmacol. 79, 301-305 (1982)

9 Lord, J.A.H., Waterfield, A.A., Hughes, J. and Kosterlitz, H.W.: Endogenous opioid peptides: multiple agonists and receptors. Nature 267, 495-499 (1977)

10 Cowan, A., Lewis, J.W. and Macfarlane, I.R.: Agonist and antagonist properties of buprenorphine, a new antinociceptive agent. Br. J. Pharmacol. 60, 537-545 (1977)

11 Dum, J.E. and Herz, A.: In vivo receptor binding of the opiate partial agonist, buprenorphine, correlated with its agonistic and antagonistic actions. Br. J. Pharmacol. 74, 627-633 (1981) 